\title{
Anatomical organization of the lateral cervical nucleus in Artiodactyls
}

\author{
Annamaria Grandis ${ }^{1}$ - Anna Gardini ${ }^{1}$ - Claudio Tagliavia ${ }^{1}$ - Giulia Salamanca ${ }^{1}$ Jean-Marie Graïc ${ }^{2}$. \\ Margherita De Silva ${ }^{1} \cdot$ Cristiano Bombardi ${ }^{1}$
}

Received: 24 February 2021 / Accepted: 5 April 2021 / Published online: 17 April 2021

(c) The Author(s) 2021

\begin{abstract}
The presence of the lateral cervical nucleus (LCN) in different mammals, including humans, has been established in a number of anatomical research works. The LCN receives its afferent inputs from the spinocervical tract, and conveys this somatosensory information to the various brain areas, especially the thalamus. In the present study, the organization of the calf and pig LCN was examined through the use of thionine staining and immunohistochemical methods combined with morphometrical analyses. Specifically, the localization of calbindin-D28k (CB-D28k) and neuronal nitric oxide synthase (nNOS) in the LCN was investigated using the immunoperoxidase method. Calf and pig LCN appear as a clearly defined column of gray matter located in the three cranial segments of the cervical spinal cord. Thionine staining shows that polygonal neurons represent the main cell type in both species. The calf and pig LCN contained CB-D28k-immunoreactive (IR) neurons of varying sizes. Large neurons are probably involved in the generation of the cervicothalamic pathway. Small CB-D28k-IR neurons, on the other hand, could act as local interneurons. The immunoreactivity for nNOS was found to be mainly located in thin neuronal processes that could represent the terminal axonal portion of nNOS-IR found in laminae III e IV. This evidence suggests that nitric oxide (NO) could modulate the synaptic activity of the glutamatergic spinocervical tracts. These findings suggest that the LCN of Artiodactyls might play an important role in the transmission of somatosensory information from the spinal cord to the higher centers of the brain.
\end{abstract}

Keywords Lateral cervical nucleus $\cdot$ Calbindin-D28k $\cdot$ Neuronal nitric oxide synthase $\cdot$ Calf $\cdot$ Pig

\section{Introduction}

The lateral cervical nucleus (LCN) is a column of gray matter located in the most caudal part of the medulla and in the dorsal portion of the lateral funiculus of the $\mathrm{C} 1, \mathrm{C} 2$, and C3 spinal segments (Rexed and Brodal 1951). This nucleus receives mechanical and noxious stimuli from the skin (including hair) through the spinocervical tract, which originates in laminae III and IV along the length

Cristiano Bombardi

cristiano.bombardi@unibo.it

1 Department of Veterinary Medical Sciences, University of Bologna, Ozzano dell'Emilia, BO, Italy

2 Department of Comparative Biomedicine and Food Science, University of Padova, Legnaro, PD, Italy of the ipsilateral spinal cord (Cervero et al. 1977; Craig and Tapper 1978; Brown et al. 1980; Craig et al. 1992; Pubols and Haring 1995), and primarily projects to the contralateral ventroposterior and posterior regions of the thalamus (Smith and Apkarian 1991; Broman and Ottersen 1992; Zhang and Broman 1998). Glutamate is the primary excitatory neurotransmitter of the somatosensory and nociceptive systems (Bhave et al. 2001). However, many physiological neuronal processes, such as signal transduction, are also regulated by other neuroactive molecules, such as calbindin-D28k (CB-D28k) and the neuronal nitric oxide synthase (nNOS), as it is in other areas of the Artiodactyl brain (Bombardi et al. 2006; Pirone et al. 2021). CB-D28k and nNOS are localized within neurons and neuropilar areas widely distributed in the dorsal, lateral and ventral horns of the spinal cord. In particular, the localization of a large amount of CB-D28k- and nNOS-immunoreactive 
(IR) neurons in the superficial layers of the dorsal horn suggests that these proteins play a role in sensory and nociceptive functions (Nazli and Morris 2000). Despite numerous studies conducted on the LCN of different mammals, only one anatomical study has been published over 50 years ago regarding someanatomical features of the LCN of Artiodactyls (Artiodactyla) (Verhaart 1970). It must be noted that the latest studies on the anatomical organization of the LCN in the different species date back to about twenty years ago. Furthermore, ruminants and suidae have large convoluted brains characterized by high level structures that can process information related to pain signals (Ballarin et al., 2016; Minervini et al. 2016; Graïc et al. 2018; Pirone et al. 2018, 2019; Corain et. al 2020; Peruffo et al. 2019; Cozzi et al. 2020). Therefore, in the present study, thionine staining combined with morphometrical analyses were used to determine the previously uncharted cytoarchitectonic characteristics of the LCN in calves (Bos taurus) and pigs ( $\mathrm{Sus}$ scrofa). In addition, the distribution of CB-D28k and nNOS immunoreactivity in the LCN was determined using the immunoperoxidase technique.

\section{Materials and methods}

\section{Animals and fixation}

The tissues employed for this study were obtained from three calves (male, friesian, body weight $312 \pm 6 \mathrm{~kg}$,) and three pigs (male, large white, body weight $168 \pm 5 \mathrm{~kg}$ ) aged 10 months. These animals were euthanized due to extraneurological disorders (overall different pathologies concerning paratopias of the digestive system). All procedures involving animals were carried out in accordance with the Italian legislation regarding experimental animals, after approval by the Ethic Scientific Committee for Experiments on Animals of the University of Bologna (Prot Rif. BQ/gf PROT 13,825-X/10 - All. 67). The animals were deeply
Fig. 1 Brightfield photomicrographs of thionine-stained coronal sections showing the topography of the bovine lateral cervical nucleus (bordered by arrowheads). The three sections are arranged from cranial to caudal direction: $\mathrm{C} 1$ (a-b), $\mathrm{C} 2$ (c-d) and $\mathrm{C} 3(\mathbf{e}-\mathbf{f})$. Abbreviations: dh, dorsal horn; lf, lateral funiculus. Scale bar $=400 \mu \mathrm{m}$ in e (applies to a, c, e) and $100 \mu \mathrm{m}$ in $\mathbf{f}$ (applies to b, $\mathbf{d}, \mathbf{f}$ )
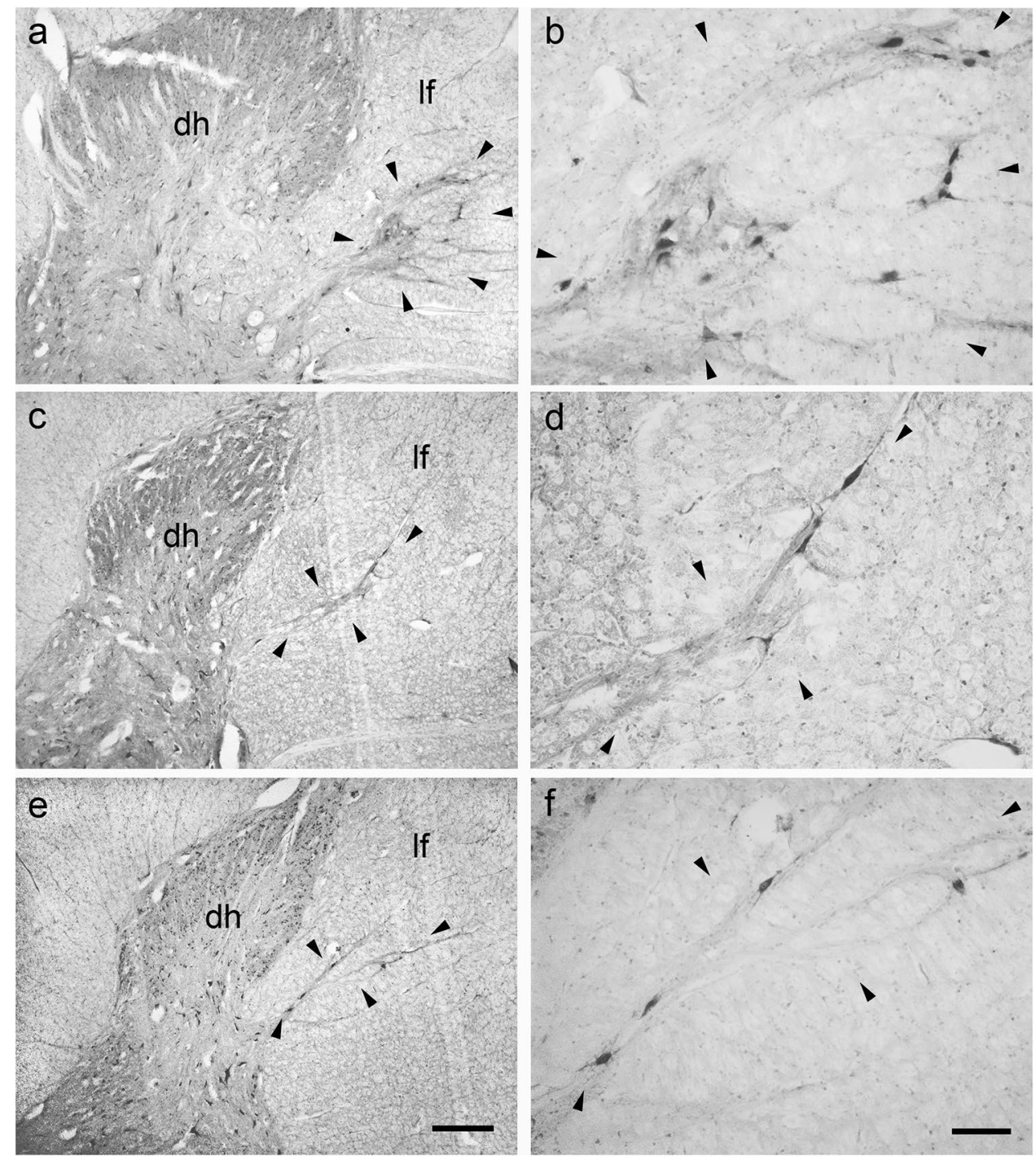
Fig. 2 Brightfield photomicrographs of thionine-stained transverse sections showing the morphology of the bovine lateral cervical nucleus neurons: polygonal (a, b), fusiform (c) and spheroidal (d). Scale bar $=20 \mu \mathrm{m}$ in $\mathrm{d}$ (applies to $\mathbf{a}-\mathbf{d})$
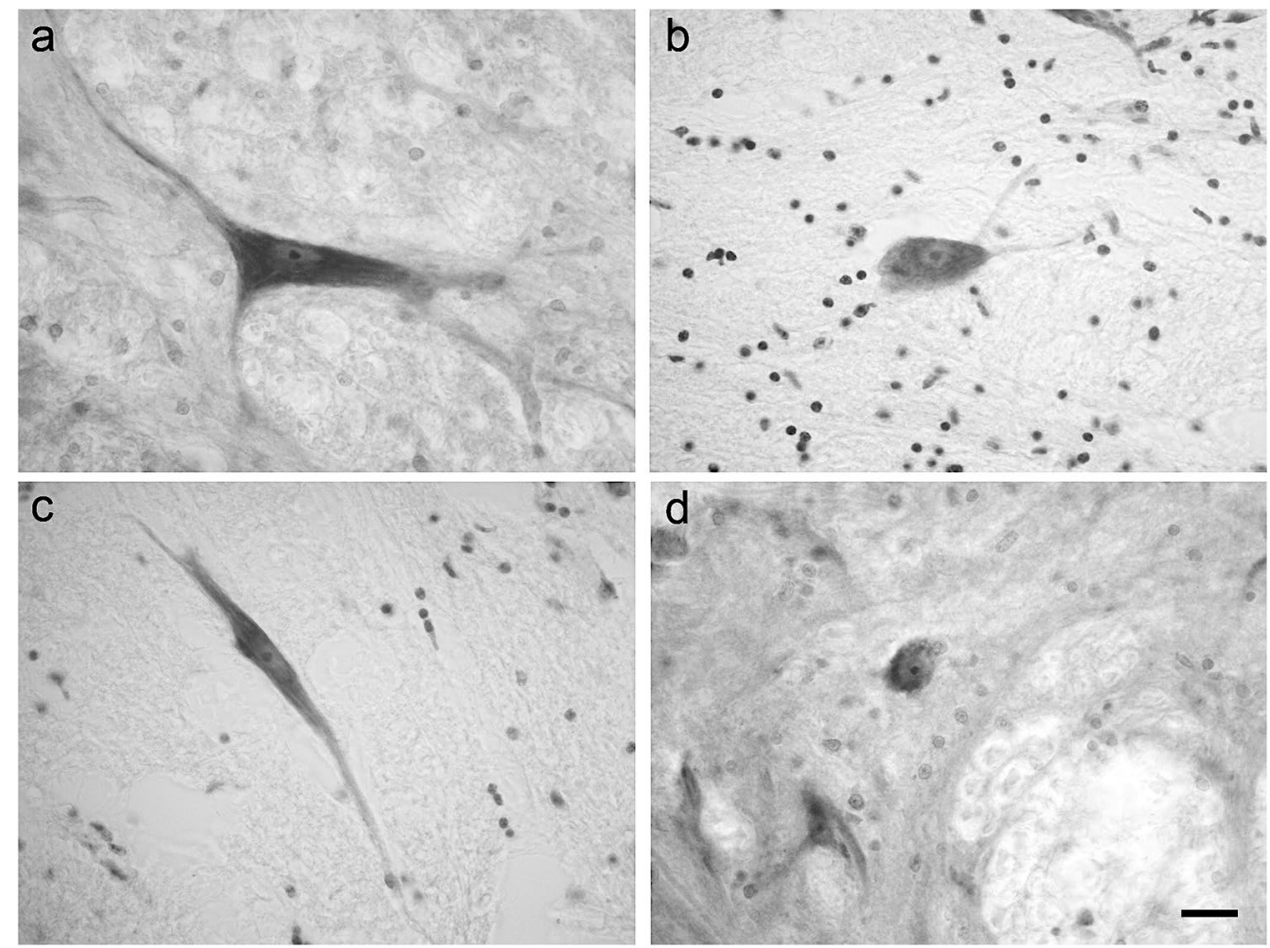

anesthetized and sacrificed through the administration of embutramide, mebenzonium iodide and tetracaine hydrochloride (Tanax). The upper spinal cord (C1, C2 and C3 spinal segments) of all the utilized subjects was immediately removed and fixed in $4 \%$ paraformaldehyde in $0.1 \mathrm{M}$ sodium phosphate buffer at $4 \mathrm{C}^{\circ}$ for $48 \mathrm{~h}$. The samples were washed in a phosphate buffer saline (PBS; $0.15 \mathrm{M} \mathrm{NaCl}$ in $0.01 \mathrm{M}$ sodium phosphate buffer, $\mathrm{pH} 7.2$ ) solution, and later stored in a PBS solution containing $30 \%$ sucrose and $0.1 \%$ sodium azide $(\mathrm{pH} 7.4) \mathrm{at}+4 \mathrm{C}^{\circ}$. Coronal sections were cut at a thickness of $40 \mu \mathrm{m}$ using a freezing sliding microtome. The sections were stored in a PBS solution containing 30\% sucrose and $0.1 \%$ sodium azide (for immunohistochemical staining) or in $4 \%$ paraformaldehyde (for thionine staining) at $+4^{\circ} \mathrm{C}$ until further processing.

\section{Immunoperoxidase experiments}

The free-floating coronal sections were collected from the PBS solution containing 30\% sucrose and $0.1 \%$ sodium azide, and washed in $0.01 \mathrm{M}$ PBS for 3 times. To eliminate endogenous peroxidase activity, the sections were treated with $1 \% \mathrm{H} 2 \mathrm{O} 2$ in PBS for $15 \mathrm{~min}$, and then washed in PBS six times. To block non-specific binding, the sections were incubated in a solution containing 10\% normal goat serum (Sigma-Aldrich, G9023, Missouri, USA) and 0.5\% Triton $\mathrm{X}-100$ in PBS at room temperature (RT) for $2 \mathrm{~h}$. Thereafter, the sections were soaked in a solution containing a rabbit anti-calbindin D-28 k polyclonal antibody (diluted 1:1000; CB-38a, Swant, Marly, Switzerland) or a mouse anti-nNOS monoclonal antibody (diluted 1:80, sc-5302, Santa Cruz Biotechology, CA, USA) at $4{ }^{\circ} \mathrm{C}$ for $48 \mathrm{~h}$. The final concentrations of the primary antibodies were established by performing immunoperoxidase reactions using different dilution patterns. The primary antibodies were diluted in an antibody diluent $(1.8 \% \mathrm{NaCl}$ in $0.01 \mathrm{M}$ sodium phosphate buffer containing $0.1 \%$ sodium azide) containing $1 \%$ normal goat serum and $0.5 \%$ Triton X-100. Afterwards, the sections were washed in PBS 3 times (10 min each). Thereafter, the sections were incubated in a solution containing a goat biotinylated anti-rabbit antibody (1:200, BA-1000, Vector Laboratories, Burlingame, CA, USA) or a goat biotinylated
Table 1 Distribution of polygonal, fusiform and spheroidal neurons in bovine lateral cervical nucleus

\begin{tabular}{lllll}
\hline Spinal segments & Polygonal neurons & Fusiform neurons & Spheroidal neurons & $\begin{array}{l}\text { Total } \\
\text { neurons } \\
\text { counted }\end{array}$ \\
\hline $\mathrm{C} 1$ & $235(77.5 \%)$ & $46(15.2 \%)$ & $22(7.3 \%)$ & 303 \\
$\mathrm{C} 2$ & $111(89.5 \%)$ & $10(8.1 \%)$ & $3(2.4 \%)$ & 124 \\
C3 & $129(96.3 \%)$ & $5(3.7 \%)$ & $0(0 \%)$ & 134 \\
Total C1-C3 & $475(84.7 \%)$ & $61(10.9 \%)$ & $25(4.4 \%)$ & 561 \\
\hline
\end{tabular}


anti-mouse antibody (diluted 1:200, BA-9200, Vector Laboratories, Burlingame, CA, USA), 1\% normal goat serum, and $0.3 \%$ Triton X-100 in PBS at RT for $2 \mathrm{~h}$. Subsequently, the sections were washed in PBS 3 times for 10 min each and were then soaked in avidin-biotin complex (ABC kit Vectastain, PK-6100, Vector Laboratories, Burlingame, CA, USA) at RT for 45 min. After another three washes in PBS, the immunoperoxidase reaction was developed in a solution containing 3-3'-diaminobenzidine (DAB kit, SK-4100, Vector Laboratories, Burlingame, CA, USA). Finally, the slides were dried overnight, dehydrated in ethanol, cleared in xylene, and coverslipped with Entellan (Merck, Darmstaldt, Germany).

\section{Antibody specificity and controls}

Using the immunoperoxidase method, no detectable immunolabeling was evidenced on the control sections, for which the primary antibodies had been omitted. The omission of the primary antibodies, as well as the replacement of the secondary antibodies with unsuited secondary antibodies resulted in the elimination of all immunohistochemical staining. In every batch, a positive control was included to ascertain the specific staining.

\section{Thionine staining}

To determine the anatomical boundaries and the cytoarchitectural features of the LCN, the sections that were adjacent to the immunoperoxidase preparations were stained with thionine, as described hereafter. Sections were removed from the $4 \%$ paraformaldehyde solution, mounted on gelatincoated slides, and dried at $37^{\circ} \mathrm{C}$ overnight. Afterwards, the sections were defatted in a mixture of chloroform/ethanol $100 \%$ (1:1) for $1 \mathrm{~h}$, and then rehydrated through a progressive series of decreasing concentrations of ethanol (3 min in $100 \%$ ethanol, 2 min in 95\% ethanol, 2 min in 90\% ethanol, $2 \mathrm{~min}$ in 80\% ethanol, $2 \mathrm{~min}$ in 70\% ethanol, $2 \mathrm{~min}$ in 50\% ethanol), immersed in $\mathrm{dH} 2 \mathrm{O}$ for $2 \mathrm{~min}$, and finally stained in a $0.125 \%$ thionine (Fisher Scientific) solution for $15 \mathrm{~min}$, dehydrated and coverslipped with Entellan (Merck, Darmstaldt, Germany).

Table 2 Bovine lateral cervical nucleus: perikaryal area $\left(\mu \mathrm{m}^{2}\right)$ of total neuronal population

\begin{tabular}{llll}
\hline Spinal segments & $\begin{array}{l}\text { Mean } \\
\text { area } \pm \text { standard } \\
\text { deviation }\end{array}$ & Minimum area & Maximum area \\
\hline C1 & $358.5 \pm 210.3$ & 33.4 & 1408.1 \\
C2 & $270.4 \pm 129.4$ & 45.1 & 591.4 \\
C3 & $252.7 \pm 135,5$ & 53.9 & 1024 \\
Total C1-C3 & $313.8 \pm 185.5$ & 33.4 & 1408.1 \\
\hline
\end{tabular}

Table 3 Bovine lateral cervical nucleus: perikaryal area $\left(\mu \mathrm{m}^{2}\right)$ of polygonal neurons

\begin{tabular}{llll}
\hline Spinal segments & $\begin{array}{l}\text { Mean } \\
\text { area } \pm \text { standard } \\
\text { deviation }\end{array}$ & Minimum area & Maximum area \\
\hline C1 & $376.8 \pm 220.3$ & 33.4 & 1408.1 \\
C2 & $268.1 \pm 131.3$ & 45.1 & 591.4 \\
C3 & $250.9 \pm 136.2$ & 53.9 & 1024 \\
Total C1-C3 & $317.2 \pm 191$ & 33.4 & 1408.1 \\
\hline
\end{tabular}

\section{Analysis of sections}

The sections, stained with thionine and with immuneperoxidase procedures, were observed with a Zeiss Axioplan microscope (Carl Zeiss, Oberkochen, Germany). A KS 300 Zeiss software (Kontron Elektronik, Germany) was used for the morphometric analysis of thionine-stained and immunostained neurons. Only the neurons showing an evident nucleus (as well as a nucleolus, in the thionine-stained sections) were counted and included in the perikaryal area analysis. These data, obtained using twelve nonconsecutive sections from each cervical segment (of each animal), were measured after having manually traced the cell body outlines. Data were expressed as mean \pm standard deviation (SD). The contrast and brightness of the figures were adjusted to resemble the appearance of the labelling seen through the microscope using Adobe Photoshop CS3 Extended 10.0 software (Adobe Systems, San Jose, CA).

\section{Results}

\section{Bovine lateral cervical nucleus: thionine staining}

A well-developed LCN was present in the dorsal part of the bovine lateral funiculus. This nucleus appeared as a longitudinal column of neurons extending from the cranial part of $\mathrm{C} 1$ to the caudal end of $\mathrm{C} 3$ (Fig. 1a-f). The calf LCN is large and elongated in transversal plane and often connected to the dorsal horn or the intermediate zone of the spinal gray matter. The LCN shifts from lateral to ventrolateral to the

Table 4 Bovine lateral cervical nucleus: perikaryal area $\left(\mu \mathrm{m}^{2}\right)$ of fusiform neurons

\begin{tabular}{llll}
\hline Spinal segments & $\begin{array}{l}\text { Mean } \\
\text { area } \pm \text { standard } \\
\text { deviation }\end{array}$ & Minimum area & Maximum area \\
\hline C1 & $296.7 \pm 163.6$ & 91.1 & 872.5 \\
C2 & $283.7 \pm 110.4$ & 148 & 436.8 \\
C3 & $299.1 \pm 116.6$ & 137.2 & 391.7 \\
Total C1-C3 & $296.1 \pm 151.8$ & 91.1 & 872.5 \\
\hline
\end{tabular}


Table 5 Bovine lateral cervical nucleus: perikaryal area $\left(\mu m^{2}\right)$ of spheroidal neurons

\begin{tabular}{llll}
\hline Spinal segments & $\begin{array}{l}\text { Mean } \\
\text { area } \pm \text { standard } \\
\text { deviation }\end{array}$ & Minimum area & Maximum area \\
\hline $\mathrm{C} 1$ & $287 \pm 160.2$ & 83.9 & 771.7 \\
$\mathrm{C} 2$ & $312.3 \pm 154$ & 178.7 & 480.8 \\
$\mathrm{C} 3$ & $/$ & $/$ & $/$ \\
Total C1-C3 & $290.1 \pm 156.4$ & 83.9 & 771.7 \\
\hline
\end{tabular}

neck of the dorsal horn (Fig. 1a-f). A total of 561 clearly visible neurons was counted in the calf LCN. Most of the LCN neurons (54\%) were located in the $\mathrm{C} 1$ spinal segment (303 C1 neurons / 561 LCN neurons). C2 and C3 contained $22.1 \%$ (124 C2 neurons /561 LCN neurons), and 23.9\% (134 C3 neurons /561 LCN neurons) of neurons, respectively. The calf LCN neuronal populations could be divided into three morphological cell types: polygonal, fusiform, and spheroidal (Fig. 2a-d). Polygonal neurons were multipolar with angular soma from which a minimum of three primary dendrites of variable thickness arose (Fig. 2a, b). The somata of fusiform cells had an ovoidal shape with primary dendrites originating from the opposite poles of the soma (Fig. 2c). Spheroidal cells had a round cell body and did not show any visible dendrite (Fig. 2d). Spheroidal neurons were not observed at $\mathrm{C} 3$ level. The distribution and the perikaryal area of the different neuronal types in the bovine LCN are reported in Tables 1, 2, 3, 4 and 5. We did not observe a clear division of the bovine LCN. However, the dorsolateral neurons appeared larger than the ventromedial ones.

\section{Porcine lateral cervical nucleus: thionine staining}

The pig LCN consisted of a longitudinally-oriented column of neurons which was less evident than that seen in calves.
Fig. 3 Brightfield photomicrographs of thionine-stained coronal sections showing the topography of the porcine lateral cervical nucleus (bordered by arrowheads). The three sections are arranged from cranial to caudal direction: $\mathrm{C} 1$ (a-b), $\mathrm{C} 2$ (c-d) and C3 (e-f). Abbreviations: dh, dorsal horn; lf, lateral funiculus. Scale bar $=400 \mu \mathrm{m}$ in e (applies to $\mathbf{a}, \mathbf{c}, \mathbf{e})$ and $100 \mu \mathrm{m}$ in $\mathrm{f}$ (applies to $\mathbf{b}, \mathbf{d}, \mathbf{f}$ )
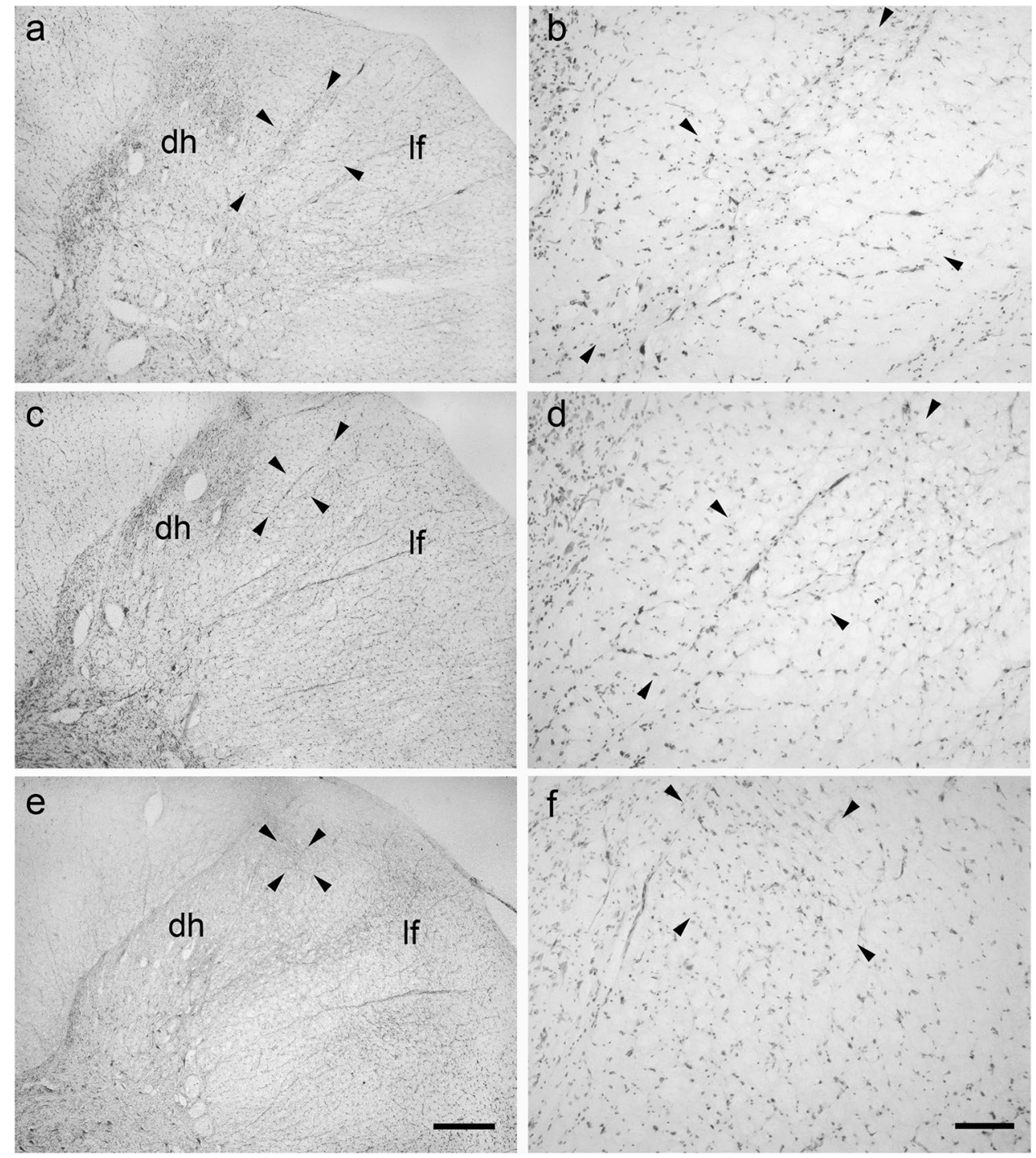
Fig. 4 Brightfield photomicrographs of thionine-stained transverse sections showing the morphology the of the porcine lateral cervical nucleus neurons: polygonal (a) and, occasionally, fusiform (b). Scale bar $=20 \mu \mathrm{m}$ in $b$ (applies to $\mathbf{a}, \mathbf{b})$
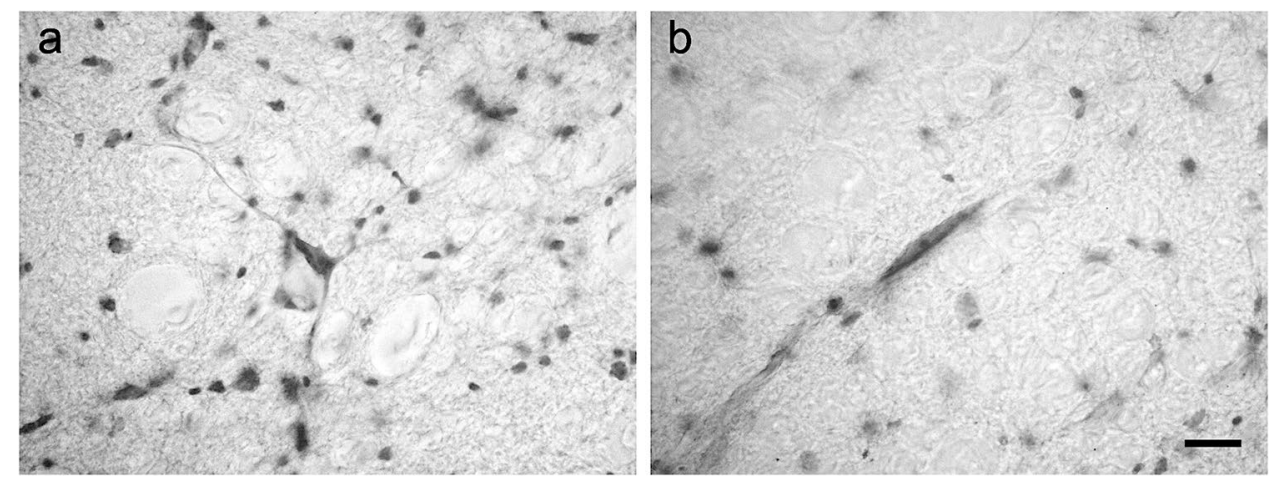

This small group of neurons was located in the dorsal portion of the lateral funiculus of the three cranial cervical spinal segments (Fig. 3a-f). As found in the calf, the LCN changes its position from lateral to ventrolateral to the dorsal horn (Fig. 3a-f). The connections between the LCN and the spinal gray matter were less consistent than those observed in calves. All porcine LCN neurons showed a polygonal shape (Fig. 4a), whereas ovoidal neurons were not evidenced. Few fusiform neurons were occasionally observed (Fig. 4b). In the pig LCN, a total of 189 clearly-visible neurons were counted in thionine preparations. As with the calf, the number of neurons decreased cranio-caudally: $\mathrm{C} 1$ contained (81/189 LCN neurons) 42.8\%, C2 (51/189 LCN neurons) $27 \%$ and C3 (57/189 LCN neurons) $30.2 \%$ of neurons. The perikaryal areas of the neuronal population in the pig LCN are reported in Table 6.

\section{Bovine lateral cervical nucleus: calbindin-D28k immunoreactive neurons}

Rare CB-D28k-IR neurons were observed throughout the entire cranio-caudal extension of the calf LCN (a total of 40 clearly-visible neurons was counted) (Fig. 5a-f). The immunostaining was especially located in the somata and primary dendrites. The CB-D28k-IR neurons were mostly located at C1 level: C1 contained (28/40) $70 \%$ of neurons, C2 (4/40) $10 \%$, and C3 (12/40) 30\%. These neurons presented

Table 6 Porcine lateral cervical nucleus: perikaryal area $\left(\mu \mathrm{m}^{2}\right)$ of total neuronal population

\begin{tabular}{llll}
\hline Spinal segments & $\begin{array}{l}\text { Mean } \\
\text { area } \pm \text { standard } \\
\text { deviation }\end{array}$ & Minimum area & Maximum area \\
\hline $\mathrm{C} 1$ & $183.1 \pm 88.4$ & 42.8 & 486.8 \\
$\mathrm{C} 2$ & $169.2 \pm 97.5$ & 44 & 516.9 \\
$\mathrm{C} 3$ & $192.3 \pm 114.1$ & 70.8 & 658.4 \\
Total C1-C3 & $182.1 \pm 99$ & 42.8 & 658.4 \\
\hline
\end{tabular}

polygonal cell bodies with clearly-evident primary dendrites (Fig. 5g). The neuropil staining was mainly caused by the expression of an immunoreactivity in the fine dendritic branching. Generally, the neurons in the LCN were less intensely immunostained than those located in the superficial layers of the dorsal horn (Fig. 5h). The perikaryal areas of CB-D28K-IR neurons in the bovine LCN are reported in Table 7.

\section{Porcine lateral cervical nucleus: calbindin-D28k immunoreactive neurons}

A moderate number (52) of CB-D28k-IR neurons was counted along the whole extension of the LCN (from C1 to C3) (Fig. 6a-f). These cells were usually polygonal with well-visible primary dendrites (Fig. 6g). As already seen in the calf, also the pig CB-D28k-IR neurons tended to decrease in a cranio-caudal direction: $\mathrm{C} 1$ contained (36/52) $69.2 \%$ neurons, C2 (8/52) $15.4 \%$, and C3 (8/52) $15.4 \%$. The LCN also contained fine dendritic processes that were immunoreactive for the CB-D28K. The neurons located in the superficial laminae of the dorsal horn were more intensely immunolabelled than those located in the LCN (Fig. 6h). The perikaryal areas of CB-D28k-IR neurons in the pig $\mathrm{LCN}$ are reported in Table 8 .

\section{Bovine and porcine lateral cervical nucleus: $\mathrm{nNOS}$ immunoreactivity}

The immunoreactivity for nNOS was observed in the LCN (from $\mathrm{C} 1$ to $\mathrm{C} 3$ ) of both species (Figs. 7a-f and 8a-f). The staining mostly consisted of multiple varicose and diffuse neuropil staining, whereas no neurons were evidenced (Figs. $7 \mathrm{~g}$ and $8 \mathrm{~g}$ ). In the laminae I-III of the dorsal horn, unlike what was observed in the LCN, immunoreactive somata were present (Figs. $7 \mathrm{~h}$ and $8 \mathrm{~h}$ ). 
Fig. 5 Brightfield photomicrographs of transverse sections showing the distribution of calbindin-D28K (CB-D28k) immunoreactivity in the bovine lateral cervical nucleus (bordered by arrowheads). The three sections are arranged from cranial to caudal direction: $\mathrm{C} 1$ (a-b), C2 (c-d) and C3 (e-f). Note that CB-IR neurons have polygonal somata from which evident primary dendrites arise (g). Generally, these neurons are less intensely immunostained than those located in superficial layers of the dorsal horn (h). Abbreviations: dh, dorsal horn; lf, lateral funiculus. Scale $\mathrm{bar}=400 \mu \mathrm{m}$ in e (applies to $\mathbf{a}$, $\mathbf{c}, \mathbf{e}), 100 \mu \mathrm{m}$ in $\mathbf{f}$ (applies to $\mathbf{b}$. d, f), $20 \mu \mathrm{m}$ in $\mathrm{g}, 40 \mu \mathrm{m}$ in $\mathbf{h}$
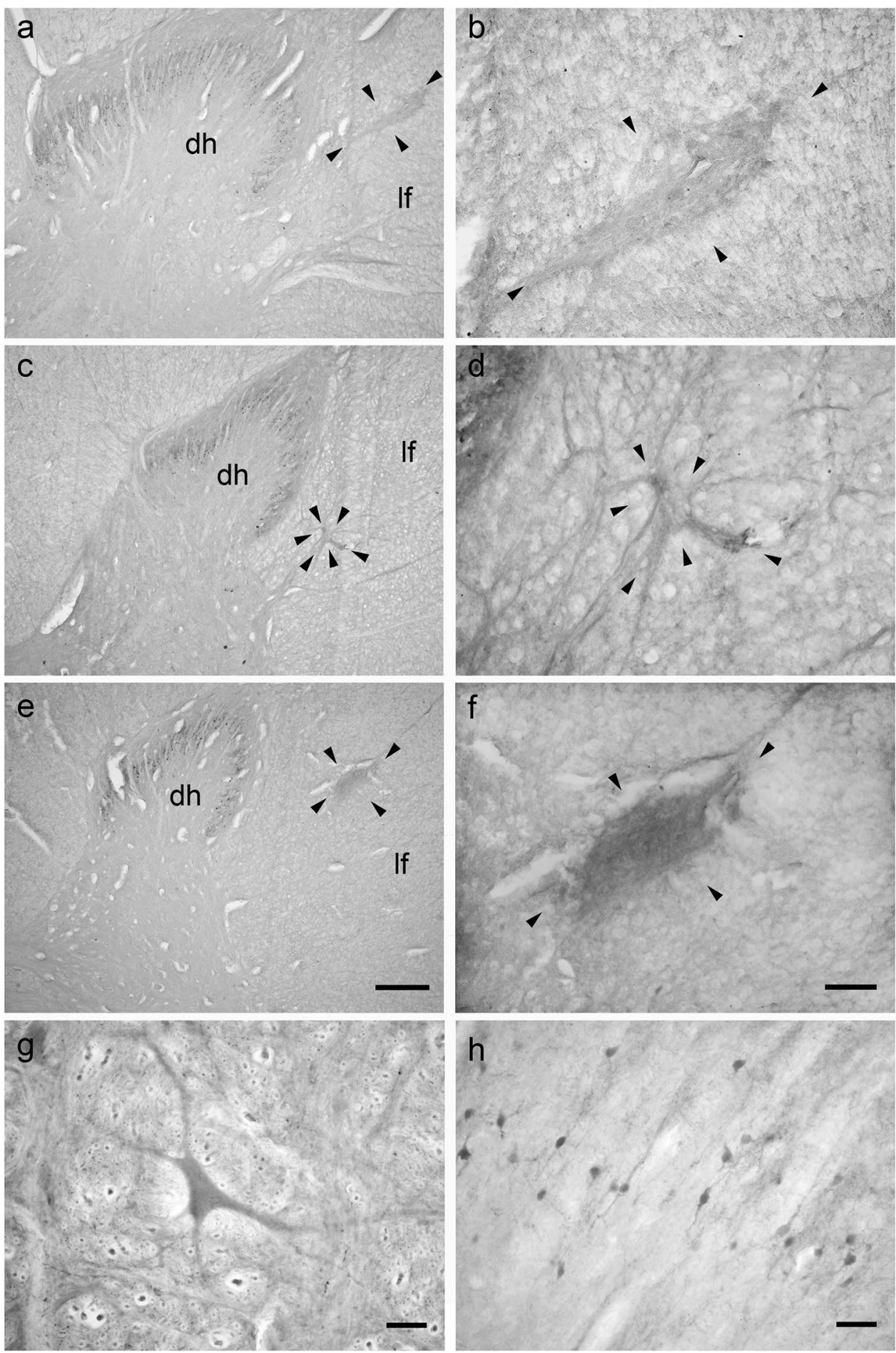
Table 7 Bovine lateral cervical nucleus: perikaryal area $\left(\mu \mathrm{m}^{2}\right)$ of total neuronal population immunoreactive for calbindin-D28k

\begin{tabular}{llll}
\hline Spinal segments & $\begin{array}{l}\text { Mean } \\
\text { area } \pm \text { standard } \\
\text { deviation }\end{array}$ & Minimum area & Maximum area \\
\hline C1 & $383.5 \pm 95.7$ & 251.9 & 559.8 \\
C2 & $558.8 \pm 197.9$ & 261.2 & 560.6 \\
C3 & $482.7 \pm 179.7$ & 355.6 & 609.7 \\
Total C1-C3 & $420.9 \pm 117.2$ & 251.9 & 609.7 \\
\hline
\end{tabular}

\section{Discussion}

Previous studies have examined the organization of the LCN in different mammalian species. These works have shown that carnivores show the most developed LCN (Giesler et al. 1987; Molander et al. 1989; Truex et al. 1970; Zhang et al. 2002). In the present study, the cytoarchitectonic organization of the bovine and porcine LCN was investigated by using a combination of thionine staining and morphometrical analyses. In addition, information regarding the distribution of the immunoreactivity for the CB-D28k and nNOS in the LCN of these species was provided. To estimate the calf and pig LCN thionine- or immunoperoxidase-stained neuronal count, we counted the neurons that contained a well-visible nucleus (as well as a nucleolus, only when analyzing thionine preparations). It should be emphasized that our primary objective was to describe the general organization of the calf and pig LCN, rather than calculate an accurate total neuronal count. Therefore, twelve nonconsecutive sections obtained from each cervical segment (of each animal) were analyzed, to obtain an anatomical characterization with general neuronal patterns without quantitative neuron counting.

In agreement with previous investigations, the results of the present study show that the bovine and porcine LCN appear as a clearly defined column of gray matter located in the three cranial segments of the cervical spinal cord. Consistently with what had been reported for carnivores, most calf and pig LCN neurons were found to be located in the rostral part of the nucleus. In general, bovine and porcine LCN neurons are morphologically similar to the neurons observed in other species (Giesler et al. 1987; Molander et al. 1989; Truex et al. 1970; Zhang et al. 2002). Interestingly, the calf LCN showed a large variability in neuronal morphology, although the prevalent cell types were polygonal, analogously to what was observed in the pig. In addition, the size of LCN neurons was larger in calves than it was in pigs. Since previous studies have demonstrated that the neuronal size of the LCN neurons is not related to the animal body size (Truex et al. 1970), we could assume that the bovine LCN is more developed than that of the pig. This is the opposite of the situation found in other nuclei in the bovine compared to the pig (Graïc et al. 2018). Further studies on neuronal morphometry could clarify this point (Grisan et al. 2018; Corain et al. 2020). The bovine and porcine LCN contained a low percentage of CB-D28k-IR cells; these presented large somata in both species, suggesting their potential involvement in the cervicothalamic pathway. Indeed, neurons containing CB-D28k located in the spinal cord can emit long ascending projections to the supraspinal area. In addition, it was noted that the injection of horseradish peroxidase (HRP) in the cat thalamus resulted in the specific labelling of the large neurons located in the LNC (Craig and Burton 1979). The small CB-D28k-IR neurons of the LCN might be interpreted as local interneurons. Consistently, small-sized GABAergic neurons were observed in the monkey and cat LCN (Broman and Westman 1988; Broman and Blomqvist 1989a; Broman and Pubols 1996). The immunoreactivity for nNOS seems to be mainly expressed by thin processes that are present in the lateral cervical nucleus. Since the spinocervical tract originates from neurons located in the dorsal horn throughout the entire length of the spinal cord, the thin processes that immunoreacted to the nNOS could represent the terminal axonal portions of the numerous nNOS-IR found in laminae III e IV (Saito et al. 1994; Bombardi et al. 2013). In such a way, nitric oxide (NO) could modulate the synaptic activity of the glutamatergic spinocervical tracts (Broman et al. 1990). The somatosensory information, ultimately destined to the neocortex, is carried to the thalamic nuclei through three different main pathways: the dorsal column-medial lemniscus, the spinothalamic tract and the spinocervicothalamic pathway (Paxinos 2015). The present study, by reporting the presence of a well-developed LCN in bovines and, to some extent, in pigs, indicates that the spinocervicothalamic pathway conveys somatosensory and noxious information from the peripheral receptors to the higher centers of the brain in these species, as well. In the monkey, cat and rat, numerous substance P (SP)immunoreactive fibers were also observed in the LCN (Giesler and Elde 1985; Broman and Blomqvist 1989b; Broman and Pubols 1993). Since SP immunoreactivity is associated with nociceptive function, our further studies will aim to determine the distribution of SP immunoreactivity in the LCN of Artiodactyls as well. By improving the knowledge regarding the nociceptive system of Artiodactyls, this study provides solid morphofunctional bases supporting the implementation of the welfare of animals, including food-producing animals. 
Fig. 6 Brightfield photomicrographs of transverse sections showing the distribution of calbindin-D28K (CB-D28k) immunoreactivity in the porcine lateral cervical nucleus (bordered by arrowheads). The three sections are arranged from cranial to caudal direction: $\mathrm{C} 1$ (a-b), C2 (c-d) and C3 (e-f). CB-D28k-immunoreactive neurons are polygonal with evident primary dendrites $(\mathbf{g})$. As in the bovine LCN, these neurons are more faintly immunostained than those of the superficial layers of the dorsal horn (h). Abbreviations: dh, dorsal horn; If, lateral funiculus. Scale bar $=400 \mu \mathrm{m}$ in e (applies to $\mathbf{a}$, c, e) and $100 \mu \mathrm{m}$ in $\mathrm{f}$ (applies to b, d, f), $20 \mu \mathrm{m}$ in $\mathrm{g}, 40 \mu \mathrm{m}$ in $\mathbf{h}$
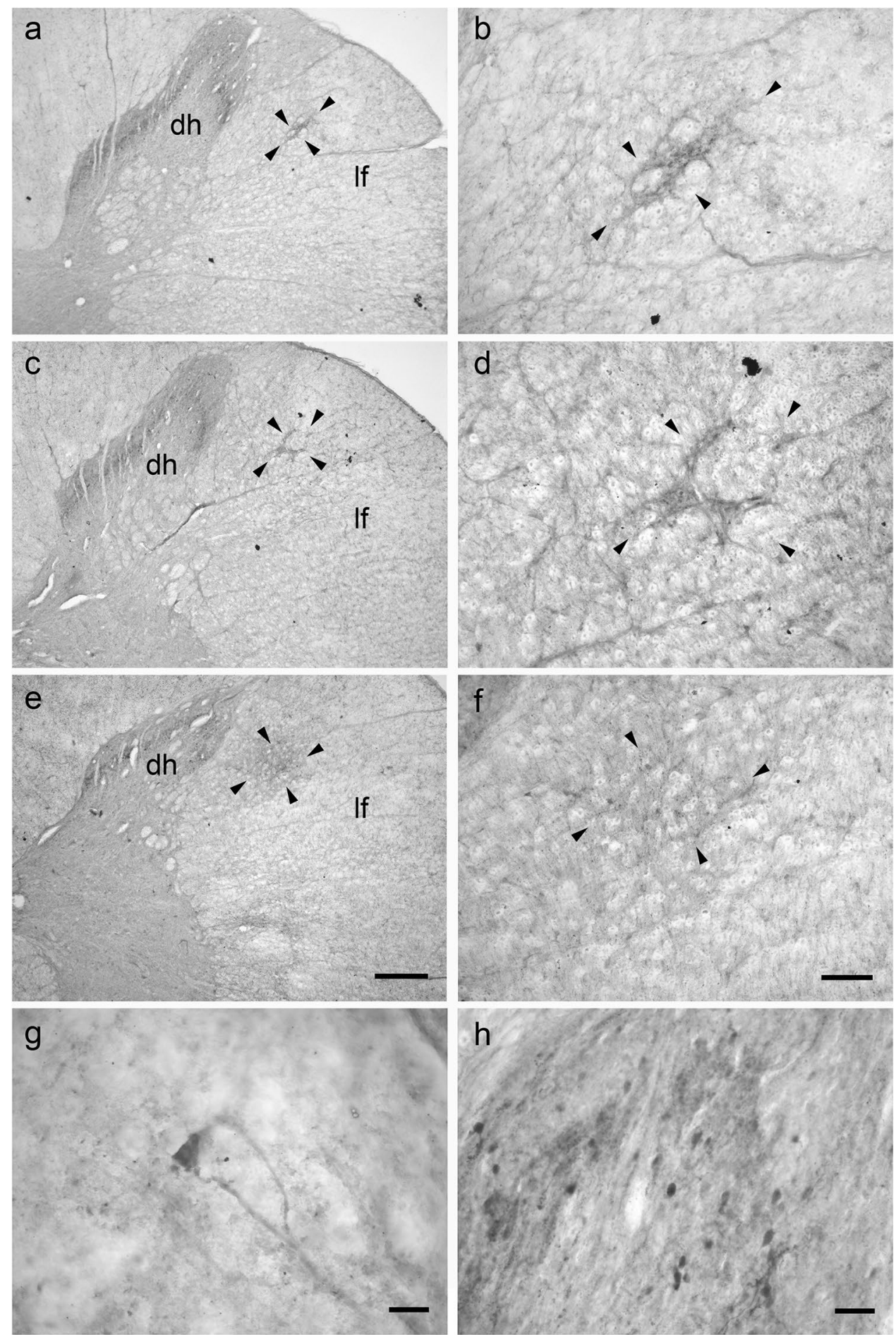
Table 8 Swine lateral cervical nucleus: perikaryal area $\left(\mu \mathrm{m}^{2}\right)$ of total neuronal population immunoreactive for calbindin-D28k

\begin{tabular}{llcc}
\hline Spinal segments & Mean area \pm standard deviation & Minimum area & Maximum area \\
\hline C1 & $184.1 \pm 45.1$ & 130.4 & 246.7 \\
C2 & $111.8 \pm 29.2$ & 91.1 & 132.4 \\
C3 & $193.9 \pm 66.7$ & 146.7 & 241.1 \\
Total C1-C3 & $174.4 \pm 50.9$ & 91.1 & 246.7 \\
\hline
\end{tabular}

Fig. 7 Brightfield photomicrographs of transverse sections showing the distribution of neuronal nitric oxide synthase (nNOS) immunoreactivity in the bovine lateral cervical nucleus (bordered by arrowheads). The three sections are arranged from cranial to caudal direction: $\mathrm{C} 1$ (a-b), C2 (c-d) and C3 (e-f). Note that the immunostaining is located only at neuropilar level (g). Superficial laminae of the dorsal horn are more intensely immunostained than LCN (h). Abbreviations: dh, dorsal horn; lf, lateral funiculus. Scale $\mathrm{bar}=400 \mu \mathrm{m}$ in e (applies to a, $\mathrm{c}, \mathrm{e}$ ) and $100 \mu \mathrm{m}$ in $\mathrm{f}$ (applies to $\mathrm{b}, \mathrm{d}, \mathrm{f}), 40 \mu \mathrm{m}$ in $\mathrm{g}, 40 \mu \mathrm{m}$ in $\mathrm{h}$
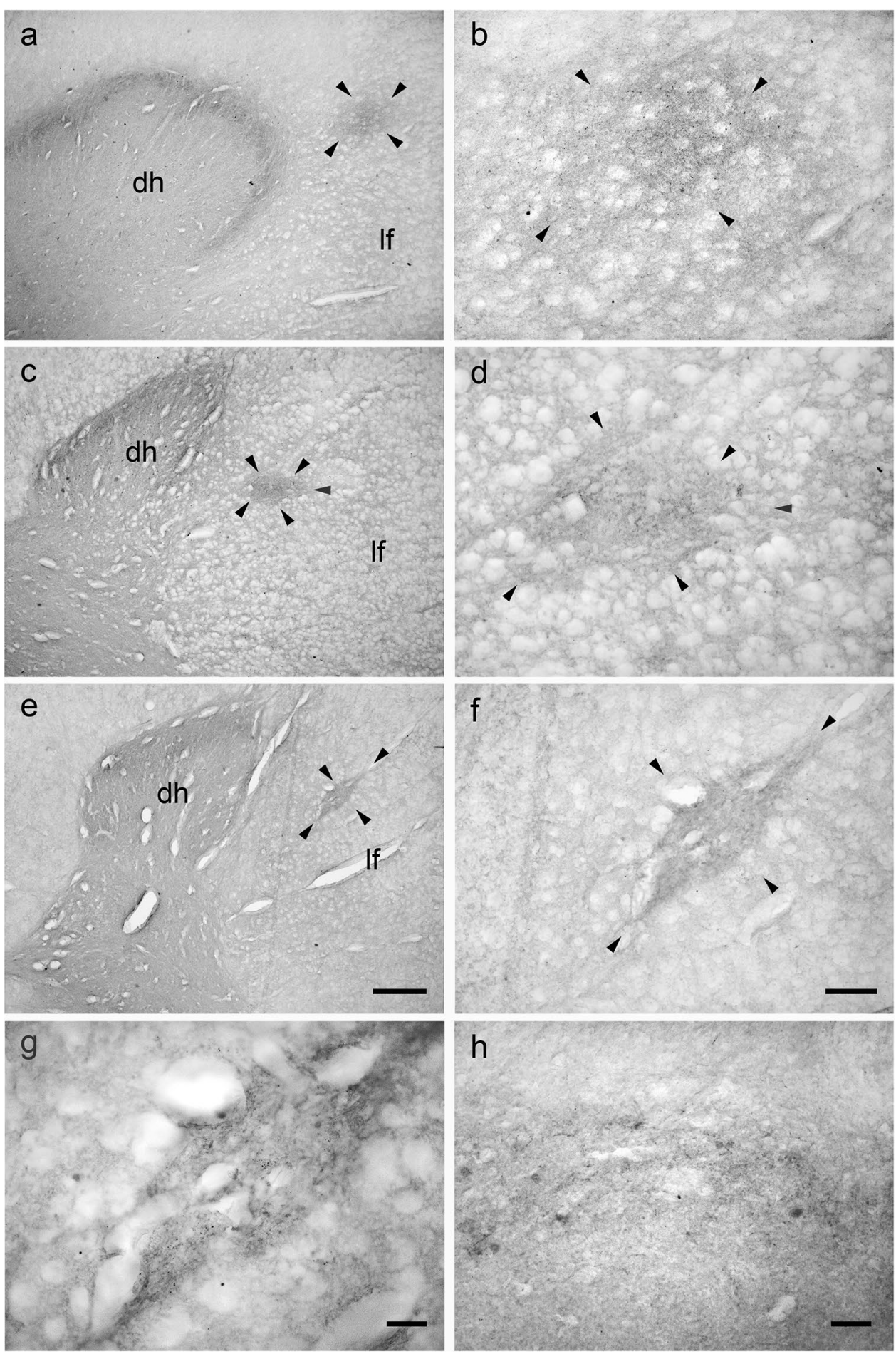
Fig. 8 Brightfield photomicrographs of transverse sections showing the distribution of neuronal nitric oxide synthase (nNOS) immunoreactivity in the porcine lateral cervical nucleus (bordered by arrowheads). The three sections are arranged from cranial to caudal direction: $\mathrm{C} 1$ (a-b), C2 (c-d) and C3 (e-f). Only neuropilar elements show immunostaining (g). Laminae I-III of the dorsal horn are more intensely immunopositive than LCN (h). Abbreviations: dh, dorsal horn; lf, lateral funiculus. Scale bar $=400 \mu \mathrm{m}$ in e (applies to $\mathbf{a}, \mathbf{c}, \mathbf{e}$ ), $100 \mu \mathrm{m}$ in $\mathbf{f}$ (applies to $\mathbf{b}, \mathbf{d}, \mathbf{f}), 40 \mu \mathrm{m}$ in $\mathrm{g}$ and $\mathrm{h}$
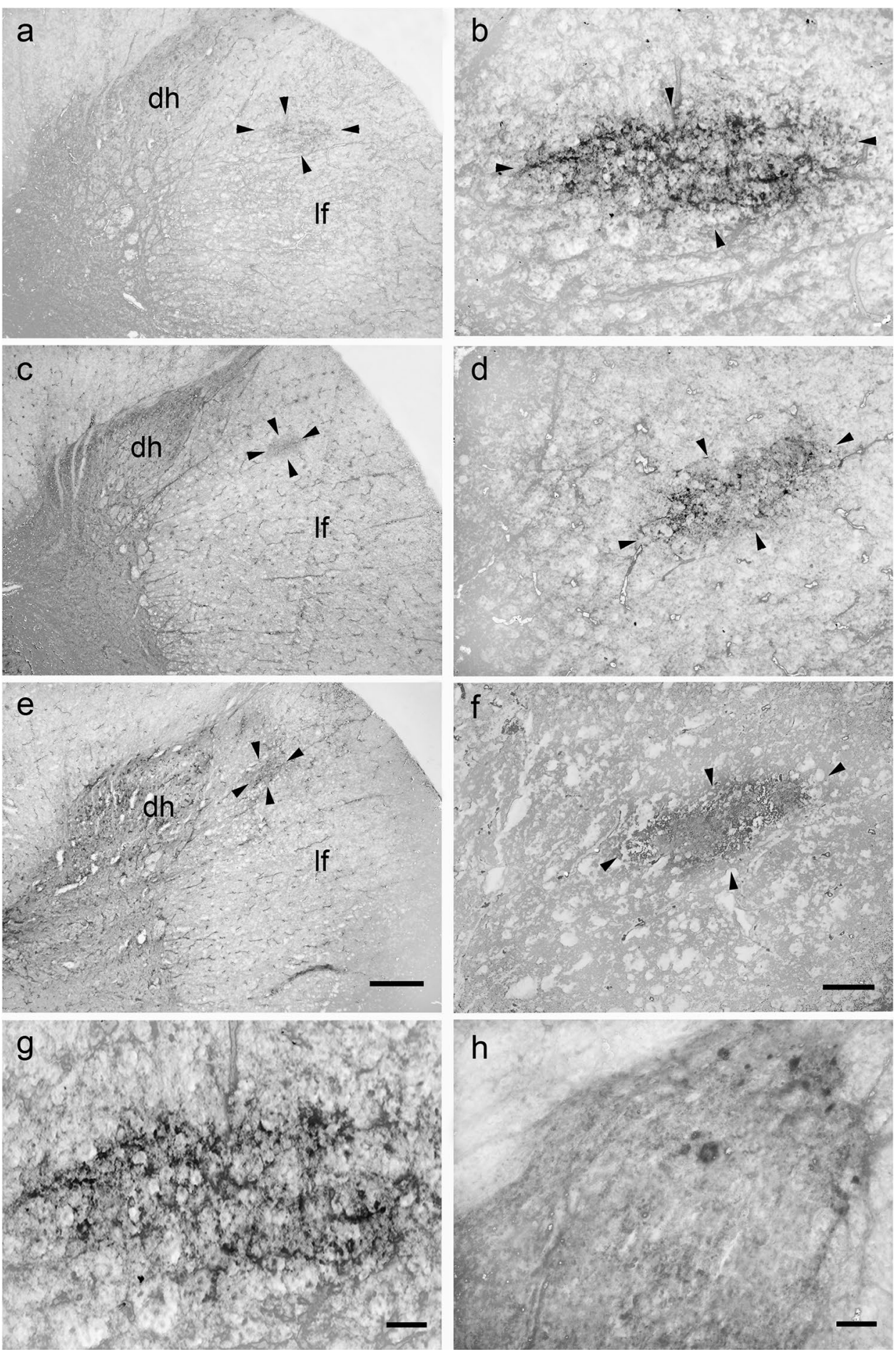
Authors' Contributions In relation with your submission Author contributions Research planning (Grandis A, Bombardi C, Gardini A), Laboratory activities (Gardini A, Bombardi C, Tagliavia C, Salamanca G, Graïc JM, De Silva M), writing - review and editing (Grandis A, Bombardi C, Graïc JM, De Silva M), Supervision (Grandis A, Bombardi C).

Funding Open access funding provided by Alma Mater Studiorum Università di Bologna within the CRUI-CARE Agreement.

Data Availability The datasets in this study are available from the corresponding author on reasonable request. All data and materials are available for publication.

\section{Declarations}

Ethical Approval The animals were euthanized due to extra-neurological disorders. All procedures involving animals were carried out in accordance with the Italian legislation regarding experimental animals, after the approval by the Ethic Scientific Committee for Experiments on Animals of the University of Bologna (Prot Rif. BQ/gf PROT 13825-X/10 - All. 67).

Consent to Participate All authors read and approved the manuscript.

Consent to Publish All authors read and approved the manuscript.

Competing Interests The authors declare that they have no conflicts of interest.

Open Access This article is licensed under a Creative Commons Attribution 4.0 International License, which permits use, sharing, adaptation, distribution and reproduction in any medium or format, as long as you give appropriate credit to the original author(s) and the source, provide a link to the Creative Commons licence, and indicate if changes were made. The images or other third party material in this article are included in the article's Creative Commons licence, unless indicated otherwise in a credit line to the material. If material is not included in the article's Creative Commons licence and your intended use is not permitted by statutory regulation or exceeds the permitted use, you will need to obtain permission directly from the copyright holder. To view a copy of this licence, visit http://creativecommons.org/licenses/by/4.0/.

\section{References}

Ballarin C, Povinelli M, Granato A, Panin M, Corain L, Peruffo A, Cozzi B (2016) The Brain of the Domestic Bos taurus: Weight, Encephalization and Cerebellar Quotients, and Comparison with Other Domestic and Wild Cetartiodactyla. PLoS ONE 11(4):e0154580. https://doi.org/10.1371/journal.pone.0154580

Bhave G, Karim F, Carlton SM, Gereau Iv RW (2001) Peripheral group I metabotropic glutamate receptors modulate nociception in mice. Nat Neurosci 4(4):417-423

Bombardi C, Grandis A, Chiocchetti R, Lucchi ML (2006) Distribution of calbindin-D28k, neuronal nitric oxide synthase, and nicotinamide adenine dinucleotide phosphate diaphorase (NADPHd) in the lateral nucleus of the sheep amygdaloid complex. Anat Embryol (Berl) 211(6):707-720. https://doi.org/10.1007/ s00429-006-0133-x

Bombardi C, Grandis A, Gardini A, Cozzi B (2013) Nitrergic neurons in the spinal cord of the bottlenose Dolphin (Tursiops truncatus). Anat Rec (Hoboken) 10:1603-1614. https://doi.org/10.1002/ar.22766
Broman J, Ottersen OP (1992) Cervicothalamic tract terminals are enriched in glutamate-like immunoreactivity: an electron microscopic double-labeling study in the cat. J Neurosci 12:204-221. https://doi.org/10.1523/JNEUROSCI.12-01-00204.1992

Broman J, Blomqvist A (1989a) GABA-immunoreactive neurons and terminals in the lateral cervical nucleus of the cynomolgus monkey. J Comp Neurol 283:415-424. https://doi.org/10.1002/ cne. 902830309

Broman J, Blomqvist A (1989b) Substance P-like immunoreactivity in the lateral cervical nucleus of the owl monkey (Aotus trivirgatus): a comparison with the cat and rat. J Comp Neurol 289:111-117. https://doi.org/10.1002/cne.902890109

Broman J, Pubols BH Jr (1993) Substance P-like and serotonin-like immunoreactivity in the lateral cervical nucleus of the raccoon. J Comp Neurol 329:354-364. https://doi.org/10.1002/cne.903290306

Broman J, Pubols BH Jr (1996) The raccoon lateral cervical nucleus: mediolateral organization of GABA-positive and GABA-negative neurons and fibers. Anat Embryol (Berl) 193:463-474. https://doi.org/10.1007/BF00185877

Broman J, Westman J (1988) GABA-immunoreactive neurons and terminals in the lateral cervical nucleus of the cat. J Comp Neurol 274:467-482. https://doi.org/10.1002/cne.902740402

Broman J, Westman J, Ottersen OP (1990) Ascending afferents to the lateral cervical nucleus are enriched in glutamate-like immunoreactivity: a combined anterograde transport-immunogold study in the cat. Brain Res 520:178-191. https://doi.org/10. 1016/0006-8993(90)91704-k

Brown AG, Fyffe RE, Noble R, Rose PK, Snow P (1980) The density, distribution and topographical organization of spinocervical tract neurons in the cat. J Physiol 300:409-428. https://doi.org/10.1113/ jphysiol.1980.sp013169

Cervero F, Iggo A, Molony V (1977) Responses of spinocervical tract neurones to noxious stimulation of the skin. J Physiol 267:537588. https://doi.org/10.1113/jphysiol.1977.sp011825

Corain L, Grisan E, Graï JM, Carvajal-Schiaffino R, Cozzi B, Peruffo A (2020) Multi-aspect testing and ranking inference to quantify dimorphism in the cytoarchitecture of cerebellum of male, female and intersex individuals: a model applied to bovine brains. Brain Struct Funct 225(9):2669-2688. https://doi.org/10.1007/ s00429-020-02147-x

Cozzi B, Bonfanti L, Canali E, Minero M (2020) Brain Waste: The Neglect of Animal Brains. Front Neuroanat. 2020;14:573934. https://doi.org/10.3389/fnana.2020.573934

Craig AD Jr, Burton H (1979) The lateral cervical nucleus in the cat: anatomic organization of cervicothalamic neurons. J Comp Neurol 185:329-346. https://doi.org/10.1002/cne.901850207

Craig AD, Tapper DN (1978) Lateral cervical nucleus in the cat: functional organization and characteristics. J Neurophysiol 41:1511-1534. https://doi.org/10.1152/jn.1978.41.6.1511

Craig AD, Broman J, Blomqvist A (1992) Lamina I spinocervical tract terminations in the medial part of the lateral cervical nucleus in the cat. J Comp Neurol 322:99-110. https://doi.org/ 10.1002/cne.903220108

Giesler GJ Jr, Elde RP (1985) Immunocytochemical studies of the peptidergic content of fibers and terminals within the lateral spinal and lateral cervical nuclei. J Neurosci 5:1833-1841. https://doi. org/10.1523/JNEUROSCI.05-07-01833.1985

Giesler GJ Jr, Miller LR, Madsen AM, Katter JT (1987) Evidence for the existence of a lateral cervical nucleus in mice, guinea pigs, and rabbits. J Comp Neurol 263:106-112. https://doi.org/10.1002/ cne.902630109

Graïc JM, Corain L, Peruffo A, Cozzi B, Swaab DF (2018) The bovine anterior hypothalamus: Characterization of the vasopressin-oxytocin containing nucleus and changes in relation to sexual differentiation. J Comp Neurol 526(17):2898-2917. https://doi.org/ $10.1002 /$ cne. 24542 
Grisan E, Graï JM, Corain L, Peruffo A (2018) Resolving single cells in heavily clustered Nissl-stained images for the analysis of brain cytoarchitecture. 2018 IEEE 15th International Symposium on Biomedical Imaging (ISBI 2018), 2018-April(Isbi), 427-430. https://doi.org/10.1109/ISBI.2018.8363608

Minervini S, Accogli G, Pirone A, Graïc JM, Cozzi B, Desantis S (2016) Brain Mass and Encephalization Quotients in the Domestic Industrial Pig (Sus scrofa). PLoS ONE 11(6):e0157378. https:// doi.org/10.1371/journal.pone.0157378

Molander C, Xu Q, Rivero-Melian C, Grant G (1989) Cytoarchitectonic organization of the spinal cord in the rat: II. The cervical and upper thoracic cord. J Comp Neurol 289:375-385. https://doi.org/ $10.1002 /$ cne. 902890303

Nazli M, Morris R (2000) Comparison of localization of the neurokinin 1 receptor and nitric oxide synthase with calbindin D labelling in the rat spinal cord. Anat Histol Embryol 29(3):141-143. https:// doi.org/10.1046/j.1439-0264.2000.00243.x

Paxinos G (2015) The Rat Nervous System, 4th edn. Academic Press London, Waltham, San Diego

Peruffo A, Corain L, Bombardi C, Centelleghe C, Grisan E, Graïc JM, Bontempi P, Grandis A, Cozzi B (2019) The motor cortex of the sheep: laminar organization, projections and diffusion tensor imaging of the intracranial pyramidal and extrapyramidal tracts. Brain Struct Funct 224(5):1933-1946. https://doi.org/10.1007/ s00429-019-01885-x

Pirone A, Miragliotta V, Ciregia F, Giannessi E, Cozzi B (2018) The catecholaminergic innervation of the claustrum of the pig. J Anat 232(1):158-166. https://doi.org/10.1111/joa.12706

Pirone A, Miragliotta V, Cozzi B, Granato A (2019) The Claustrum of the Pig: An Immunohistochemical and a Quantitative Golgi Study. Anat Rec (Hoboken) 302(9):1638-1646. https://doi.org/ 10.1002/ar.24073
Pirone A, Graï JM, Grisan E, Cozzi B (2021) The claustrum of the sheep and its connections to the visual cortex. J Anat 238(1):1-12. https://doi.org/10.1111/joa.13302

Pubols BH Jr, Haring JH (1995) The raccoon spinocervical and spinothalamic tracts: a horseradish peroxidase study. Brain Res Brain Res Rev 20:196-208. https://doi.org/10.1016/0165-0173(94)00012-e

Rexed B, Brodal A (1951) The nucleus cervicalis lateralis. A spinocerebellar relay nucleus J Neurophysiol 14:399-407. https://doi.org/ 10.1152/jn.1951.14.5.399

Saito S, Kidd GJ, Trapp BD, Dawson TM, Bredt DS, Wilson DA, Traystman RJ, Snyder SH, Hanley DF (1994) Rat spinal cord neurons contain nitric oxide synthase. Neuroscience 59:447-456. https:// doi.org/10.1016/0306-4522(94)90608-4

Smith MV, Apkarian AV (1991) Thalamically projecting cells of the lateral cervical nucleus in monkey. Brain Res 555:10-18. https:// doi.org/10.1016/0006-8993(91)90853-n

Truex RC, Taylor MJ, Smythe MQ, Gildenberg PL (1970) The lateral cervical nucleus of cat, dog and man. J Comp Neurol 139:93-104. https://doi.org/10.1002/cne.901390106

Verhaart WJ (1970) Comparative anatomical aspects of the mammalian brain stem and the cord. Stud Neuroanat 2(9):1-312

Zhang M, Broman J (1998) Cervicothalamic tract termination: a reexamination and comparison with the distribution of monoclonal antibody Cat-301 immunoreactivity in the cat. Anat Embryol 198:451-472. https://doi.org/10.1007/s004290050196

Zhang M, Liu Y, Broman J (2002) Organization of the ferret lateral cervical nucleus and cervicothalamic tract. Somatosens Mot Res 19:36-48. https://doi.org/10.1080/08990220120113039

Publisher's Note Springer Nature remains neutral with regard to jurisdictional claims in published maps and institutional affiliations. 